\title{
Adaptación de la metodología de "observación participante» al estudio de los gabinetes de prensa como fuentes periodísticas
}

\author{
Carlos Elías \\ Profesor Titular Interino de Periodismo Especializado \\ Universidad Carlos III de Madrid \\ celias@hum.uc3m.es
}

\section{INTRODUCCIÓN}

Los medios de comunicación españoles, y en general de todos los países, son cada día más dependientes de los gabinetes de prensa. Una investigación cuantitativa llevada a cabo en la Universidad del País Vasco en cinco diarios -El País, Abc, El Correo Español, Deia y Egin- demostró que si en 1978 en $30 \%$ de las noticias publicadas por estos periódicos procedía de un gabinete de prensa; 14 años después, en 1992, este porcentaje se había incrementado al 52\% (Ramírez, 1995). A principios de este siglo XXI ya hay quien sugiere que el porcentaje ha alcanzado el $80 \%$. Sea como fuere, lo importante es que cada día se hace más necesario aplicar las metodologías de investigación usadas en otras ciencias sociales como la pedagogía, la antropología y, sobre todo, la sociología a las ciencias de la información.

No obstante no existen muchas investigaciones en España que analicen, siguiendo una estricta metodología, el fenómeno de los gabinetes de prensa como fuentes de información. Este artículo expone por qué se usó esa metodología, cuáles fueron los resultados cuando se aplicó y por qué se recomienda que se utilice en más casos de investigación de gabinetes de prensa para determinar si las conclusiones que aquí se exponen pueden ser extrapolables.

En este estudio se seleccionó una fuente (y su gabinete de prensa) que fuera muy importante desde el punto de vista periodístico $\mathrm{y}$, después, investigarla a fondo. Metodológicamente se eligió el periodismo científico porque a priori parecía el más aséptico y, por tanto, los resultados también podían ser más fácilmente extrapolables: si en ciencia se manipula, se podía afirmar que en política o en economía, la manipulación, que ya se intuye, es aún mayor. 
Desde que se comenzó a plantear esta investigación sobre los gabinetes de prensa como fuentes periodísticas se vio claro que el aspecto más importante, para que la investigación tuviera éxito, era la metodología.

Preferimos una metodología cualitativa, pues los últimos estudios en esta materia sugerían que la metodología cuantitativa no suele dar una idea real del sistema. Es, por tanto, este tipo de metodología cualitativa en su aplicación a la investigación de las ciencias sociales y, en concreto, a los estudios sobre comunicación de masas, la que se describirá en este artículo haciendo hincapié en la fórmula de observación participante.

Sin embargo, debido a que no contábamos con datos numéricos de partida, tuvimos que elaborar un estudio estadístico, para el cual aplicamos algunas de las características de la metodología cuantitativa en cuanto al tratamiento de datos. Este estudio era necesario para demostrar que el gabinete de prensa elegido, el del CSIC, era relevante desde el punto de vista de las fuentes en el periodismo científico español (Elías, 2001a). En este análisis cuantitativo se seleccionaron 3.978 informaciones científicas publicadas durante 1998 en prensa española de tirada nacional. Este estudio concluyó que la fuente española más relevante en periodismo científico era el CSIC. Aparecía en el $31,1 \%$ de todas las noticias científicas y en el $48,1 \%$ de las que tenían como fuente a un español. Si se tiene en cuenta que el CSIC sólo tiene el $4,25 \%$ de los investigadores y el $16 \%$ de la producción científica, se observa la gran importancia del CSIC como fuente. Es decir, su comportamiento como fuente afecta a casi la mitad de las noticias sobre temática científica. Debe mencionarse que las universidades españolas, que aportan el $77 \%$ de la producción científica, apenas salen en los medios de comunicación.

Por tanto, el estudio del CSIC como fuente es fundamental. Teniendo en cuenta que este organismo está fuertemente centralizado y que, como organismo político y gubernamental, toda información que produzca hacia los medios de comunicación debe pasar antes por el gabinete de prensa, resultaba acertado el estudio de este gabinete. Si se hacía con una metodología adecuada las conclusiones podrán extrapolarse al resto de los gabinetes de prensa $y$, en general, a muchas otras fuentes. Los gabinetes de prensa, considerados por muchos como uno de los cánceres del periodismo actual ${ }^{1}$, constituyen, como se ha mencionado, uno de las fuentes periodísticas fundamentales en estos momentos. Sin embargo, son pocos los estudios científicos sobre ellos que han aplicado verdaderas metodologías de las ciencias sociales. Por ello, muchas conclusiones que se enseñan en las facultades de periodismo sobre estos gabinetes proceden más de juicios de valor o experiencias profesionales concretas que de estudios científicos serios.

En resumen, necesitábamos adaptar las metodologías habituales usadas en otras ciencias sociales para investigar a los gabinetes de prensa como fuentes periodísticas.

1 Véase, entre otros: Collon, M. (1999, novena edición), ;Ojo con los media! Editions EPO. Bruselas. 


\section{PRINCIPIOS TEÓRICOS DE LA METODOLOGÍA CUALITATIVA UTILIZADA}

En el pasado, una crítica frecuente a la investigación cualitativa ha sido la ausencia de procedimientos de investigación explícitos. La explicación y la claridad son importantes en todas las fases del estudio y daba la impresión, a principios del siglo $\mathrm{xx}$, de que esta metodología no aclaraba de forma adecuada sus procedimientos.

Desde el punto de la evolución histórica de la metodología cualitativa debe mencionarse que el primer periodo comenzó a mitad de los años sesenta y duró más o menos un decenio. En esta primera etapa los análisis se centraron en comparaciones con la metodología cuantitativa, sobre todo, en torno a temas como la validez, la fiabilidad o los procedimientos de muestreo de ambas.

No debe olvidarse que las metodologías cuantitativas basadas en el uso de métodos positivistas que pretenden estudiar las ciencias sociales con los parámetros de las ciencias físicas -es decir, basándose exclusivamente en la medida numérica de ciertos parámetros- tuvieron su auge en los años 30 , aunque ejercieron su influencia hasta medidos de los 60 . Los estudios basados en metodologías cuantitativas se afianzaron, como dije, en los años 30 , coincidiendo con una etapa en la que los sociólogos empezaron a dirigir su atención a los métodos de investigación tal y como se utilizan en las ciencias naturales o, para ser más precisos, teniendo en cuenta el modo en el que se percibía a la ciencia natural desde la perspectiva de la ciencia social en ese momento histórico. Los sociólogos querían emular el modelo seguido por la física para que los estudios sociales fueran considerados una ciencia. Se establecieron así ciertos diseños experimentales a la vez que el razonamiento hipotético deductivo, los cuales dominaron el pensamiento investigador, y las metodologías subrayaron el uso de técnicas de recogida de datos «objetivos» así como la estandarización de los procedimientos analíticos.

Sin embargo, a partir de los años 60 , y después de tres décadas $-30,40$ y 50 - de absoluto dominio de las metodologías cuantitativas y del funcionalismo, los científicos sociales empezaron a cuestionar el método cuantitativo para investigar las ciencias sociales. Una de las criticas más duras la producía los limitados planteamientos que tiene la estadística a la hora de evaluar el complicado comportamiento humano. Ya en 1954, Herbert Blumer, socí́logo que había pertenecido a la escuela de Chicago, considerada como la consolidadora del sistema cuantitativo, llegó a decir en un artículo publicado en la American Sociological Review que no se podía reducir la existencia social a variables numéricas.

Se deshacía así el empirismo dominante en la investigación de ciencias sociales. En 1978, Gitlin criticó duramente los métodos cuantitativos en el campo de investigación de los medios de comunicación de masas. Puede asegurarse, incluso, que Gitlin escogió las investigaciones en medios de comunicación como paradigma para demostrar que los resultados obtenidos de exclusivamente datos empíricos son erróneos desde el punto de vista meto- 
dológico. Sus trabajos contaron con numerosos adeptos pero también con bastantes críticos. Puede concluirse que esta primera etapa del desarrollo de los métodos cualitativos modernos finalizó a principios de los años 70 y se definió, básicamente, por la defensa de los presupuestos cualitativos frente a los cuantitativos.

El segundo periodo, que abarca la década de los 70 , se caracteriza por la ya absoluta preponderancia de la investigación cualitativa frente a la cuantitativa. En esta segunda etapa, superado el debate entre el método cualitativo o cuantitativo a favor del primero, se puso un mayor énfasis en la mecánica del trabajo de campo, definiendo por primera vez la metodología de observación participante o «newsmaking» así como la dirección de entrevistas abiertas. Se establecían estas dos premisas —observación participante o participativa y entrevistas abiertas - como el engranaje fundamental de la metodología cualitativa y se sostenía, ya de forma mayoritaria, que esta metodología era la idónea para abordar la investigación de la realidad de los medios de comunicación. Estas formas de investigar y las premisas concretas en las que se basaban fueron descritas en libros de texto ya clásicos de la sociología como son el de Schatzman y Strauss publicado en 1971 y el de Johnson, en 1975.

El tercer periodo, que es en el que nos encontramos en el momento de proyectar esta investigación sobre las fuentes periodísticas y, en especial, sobre los gabinetes de prensa, comenzó en los años 80. Desde el punto de vista de la investigación de esta metodología, la mayoría de los trabajos se centran en la investigación de la toma, el análisis y la interpretación de los datos. También se consolida la metodología de la observación participante como una de las modalidades más idóneas para investigar todos los aspectos relacionados con los emisores dentro del sistema de comunicación social.

\section{¿POR QUÉ CONVIENE USAR LA METODOLOGÍA DE LA OBSERVACIÓN PARTICIPANTE PARA INVESTIGAR A LAS FUENTES PERIODÍSTICAS?}

La definición más citada que explica en qué consiste esta metodología es la propuesta por Becker y Geer en 1957. Para ellos,

«Por observación participante entendemos aquel modelo en el cual el observador participa en la vida cotidiana de la gente que está siendo objeto de estudio, sea de forma abierta en el papel de investigador, sea de modo encubierto o disfrazado, observando las cosas que ocurren, escuchando lo que se dice y haciendo preguntas a la gente a lo largo de un periodo determinado de tiempo». (Becker y Geer, 1957: 39)

De acuerdo con esta definición, el objetivo principal de la investigación mediante la metodología de observación participante es la descripción, en términos fundamentales, de distintos hechos, situaciones y acciones que suceden en un escenario social concreto. 
Más tarde, en 1969, los investigadores McCall y Simmonds concretaron de forma más clara todos los métodos a utilizar en la observación participante. Según estos sociólogos:

«Probablemente es confuso considerar que la observación es un método simple (...) se refiere a una mezcla característica o combinación de métodos y técnicas que se emplea en el estudio de ciertos tipos de materias: sociedades primitivas, subculturas marginales, organizaciones complejas, movimientos sociales o grupos informales, entre otros.» (McCall y Simmonds, 1969)

Para McCall y Simmonds esta metodología implica utilizar una cierta cantidad de interacción auténticamente social en el campo al que pertenece el objeto de estudio, algunas observaciones directas de hechos conexos, algunas entrevistas formales y muchas informales, algo de cálculo sistemático, recopilación de documentos y artefactos, y apertura en la dirección que toma el estudio.

Todos estos requisitos los hemos tenido en cuenta a la hora de estudiar el sistema "el CSIC como fuente informativa», como detallaré más adelante.

Para Jankowsky y Wester, uno de los problemas de esta última definición es la inclusión de casi todo tipo de forma de interpretación y recopilación de datos bajo el encabezamiento de observación participante. De aquí, señalan los investigadores, que se vuelva difícil la diferenciación y, lo que es más importante, la comparación y evaluación de los descubrimientos que diferentes métodos de recogida de datos pueden generar. Sin embargo, Jankowsky y Wester afirman de forma rotunda que:

«La observación participante es más idónea, si la comparamos con los diseños analíticos o experimentales, para la investigación interpretativa sobre la interacción social a partir de la perspectiva de la gente que se ve implicada (Jensen y Jankowsky, 1993: 77)».

El sociólogo italiano Mauro Wolf, quien considera que esta metodología de observación participante es la más adecuada para estudiar la sociología de los emisores en el proceso informativo, indica que los datos de estudio necesarios en la metodología de observación participante deben ser recogidos por el investigador estando presente en el ambiente objeto de estudio, bien con la observación sistemática de todo lo que pasa, bien a través de conversaciones más o menos informales y ocasionales o bien con verdaderas y propias entrevistas, llevadas a cabo con los que desarrollan los procesos productivos.

«Los criterios específicos - afirma Wolf- que rigen la recogida y la estructuración del material de observación progresivamente acumulado pueden ser varios: lo importante es que la fase de observación (es decir, la presencia del investigador en el campo) esté siempre ligada a hipotesis de investigación, orientada según presupuestos teóricos precisos, y no sea indiferenciada y casual. La observación se desarrolla por tanto entre dos límites que podrían marcar su fracaso: por un lado, la insignificancia y la 
ausencia de un proyecto de investigación; por otro lado, en el polo opuesto, la imposición de una selección rígida del material observable (Wolf, 1987: 212)».

Wolf critica el hecho de que la mayoría de las investigaciones en el campo de la comunicación de masas se hayan centrado en el análisis de la información y de los receptores, pero no han llegado al fondo de la sociología de los emisores, quienes son, básicamente, los productores de noticias.

Era obvio que en nuestro empeño del estudio de las fuentes periodísticas había que utilizar esta metodología para investigar cómo es verdaderamente un gabinete de prensa como el del CSIC - una organización compleja, por lo que debíamos utilizar esta metodología según McCall y Simmonds- así como el flujo informativo que se genera a partir de la interacción entre científicos, periodistas y políticos - por lo que también, según Jankowsky y Wester, era imprescindible utilizar el método de observación participanteComo además se trataba de analizar el CSIC como emisor, debíamos según Wolf, utilizar esta metodología de observación participante.

Sin embargo, desde el punto de vista tanto teórico como práctico esta metodología tiene sus peligros. Wolf señala como uno de ellos el fenómeno del «going native» que le puede entrar al investigador mientras realiza su trabajo y que puede condicionar su «observación científica» del sistema en estudio. Y es que, también, respecto a la forma con la que el estudioso se comporta en la escena social que analiza se pueden dar numerosas variaciones. Así, puede adoptar desde una actitud de observador pasivo que reduce al mínimo las interacciones con los sujetos analizados, hasta una actitud más participativa e integrada. Pero, según Wolf, tarde o temprano, en cualquier caso, el observador se encuentra frente a un momento en el que su papel amenaza confundirse con el del «participante-de-pleno-derecho» de la actividad observada: es ésta la fase conocida como «going native». Este error, que según Wolf desvirtúa toda la investigación y sus resultados, es inherente a todas aquellas investigaciones elaboradas por miembros -trabajadores con contrato- de un gabinete de prensa sobre su propio gabinete o sobre actividades o aspectos relacionados con la actividad profesional del mismo.

Para Wolf el efecto «going native» supone que el investigador «empieza a reconocer los valores y las acciones de forma tan clara que se le hace difícil imaginar que pudieran ser distintos» (Wolf, 1996: 212), por lo que no habrá una crítica. En este contexto, el investigador asimila la forma de proceder, de pensar y hasta de valorar de los periodistas y se convierte en uno de ellos, modificando su propio papel en la situación. Sin ser un participante en sentido estricto en el proceso de producción de las noticias, es algo más que un observador. Según todos los investigadores, entre ellos Wolf, Elliot y Schlesinger, este fenómeno coincide parcialmente con la socialización a la que es sometido el científico social desde y hacia el grupo estudiado.

La interrupción o la conclusión del periodo de trabajo de campo permiten lo que Schlesinger ha definido «disengagement», una fase que normalmente 
va acompañada de la reestructuración del material recogido, reorganizándolo desde el nivel fenomenológico de las observaciones dispersas y fragmentadas al más general de las interpretaciones sociológicas sobre las características fundamentales de los procesos sociales estudiados.

Existe otro momento delicado en esta metodología y es el que corresponde al comienzo del periodo de observación, o cuando se permite el acceso al aparato periodístico, sólo durante un brevísimo periodo de tiempo (dos o tres días) lo cual es un tiempo evidentemente insuficiente, incluso para familiarizarse con el ambiente. Los resultados obtenidos de periodos de tiempo tan cortos también pueden tener defectos debidos a fallos intrínsecos a la metodología cuando los periodos de observación son excesivamente breves.

El problema de obtener acceso prolongado a las redacciones o gabinetes de prensa es evidentemente esencial para este tipo de estudios, señala Schlesinger, para quien también es muy importante que la incorporación del investigador al proceso productivo se efectúe de forma que no ocasione, ni siquiera con su mera presencia, molestias o trabas en los procesos productivos. Este debe llevarse a cabo como si el investigador no existiera o no estuviera escudriñando todas las acciones, de forma que los actores del proceso no modifiquen en nada sus actuaciones normales. En esta premisa se basa la modalidad de investigación participativa en la que el científico realiza sus averiguaciones de incógnito.

Existe otro peligro inherente a esta metodología y que es consustancial al hecho evidente de llegar al proceso de producción normalmente en etapas muy posteriores a su comienzo real. Cuando el investigador llega al campo de estudio, el proceso se encuentra en la fase de automatismo en la cual todo el procedimiento se centra en conductas y técnicas rutinarias y monótonas. Estas conductas no dejan penetrar o enmascaran las verdaderas relaciones sociológicas que llevaron a adquirir tales rutinas y que son uno de los objetos de investigación del científico social.

Para minimizar este error, según Schlesinger, el trabajo de campo debe realizarse en un periodo de tiempo en el que el proceso de producción entre en una situación de las denominadas "crisis informativa». El sociólogo estadounidense asegura que la existencia de estos momentos de crisis corrige cualquier imagen del proceso como algo automático. Según sus investigaciones, el momento de una crisis informativa implica el replanteamiento y la corrección de las rutinas y los elementos integrantes del proceso de producción de la información. Aparecen también nuevas relaciones entre los elementos de ese proceso, todo lo cual, a su juicio, matiza y corrige las observaciones llevadas a cabo durante la etapa de proceso rutinario.

\section{CRITERIOS DE ADAPTACIÓN A UN CASO CONCRETO: EL GABINETE DE PRENSA DEL CSIC}

Una vez elegido el tema a investigar - los gabinetes de prensa como fuentes (en concreto el del CSIC) - y la metodología a aplicar - la observación 
participante- quedaba por definir las pautas de esa metodología para este caso concreto.

Para cumplir las estrictas normas que exige esta metodología era imprescindible acceder al gabinete de prensa del CSIC, si se quería efectuar una investigación de su proceso de producción. Se podía haber solicitado un permiso de investigación en él, pero en el supuesto caso de que lo concedieran, la metodología quedaría invalidada por lo que Schlesinger considera «trabas al normal proceso productivo, puesto que los actores del mismo cambian sus pautas de comportamiento al sentirse investigados».

Era obvio que, también, de esa manera se tenía acceso a menos cantidad de información, pues sólo se le revelaría al investigador aquella considerada como «políticamente correcta» y había que tener en cuenta que uno de los principales problemas con los que se han encontrado los investigadores a la hora de evaluar el verdadero efecto de los gabinetes de prensa es la opacidad de los mismos y la gran capacidad que tienen para enmascarar determinados procesos.

También estaba la posibilidad de acceder en forma de trabajador contratado, pero esta modalidad, que se podía haber intentado, pues buscaban a una persona que se incorporó en febrero de 1998, invalidaría también los resultados obtenidos, pues sería contraria a la metodología de observación participante según Wolf, quien sostiene que para que ésta sea efectiva el investigador jamás debe ser «participante de pleno derecho» en el proceso de producción. Además estaba el hecho «éticamente» inaceptable de desvelar públicamente secretos obtenidos como consecuencia de haber pertenecido a una empresa a la que se critica a posteriori sus métodos de producción.

Desechadas las anteriores posibilidades, sólo quedaba el resquicio de obtener una de las dos becas que anualmente convocaba el CSIC en colaboración con la agencia Efe, para formación en periodismo científico. Esta beca permitía trabajar para formarse en periodismo científico en el gabinete de prensa del CSIC. Los becarios tenían acceso a mucha información y, además, no despertaban sospechas de que efectuara ningún tipo de investigación -pues su habitual ambición era quedarse como trabajador del gabinete-, por lo que, dando la impresión de que se hacían méritos, se podían mover bien por todo el sistema. Todo nuestro proyecto giraba en torno a la consecución de una de las dos becas a las que se presentan más de 500 personas en cada convocatoria.

Pero para que se cumplieran todas las premisas de la metodología, según Wolf, también era necesario elaborar un proyecto de investigación previo a la fase de observación participante. Además, y dado que esta investigación iba a ser objeto de una tesis doctoral que debía ser evaluada y calificada por un tribunal, este proceso debería estar absolutamente certificado. Este proceso de certificación previo nos parece fundamental.

Así, por ejemplo, para este caso concreto, en la Universidad de La Laguna figuran las certificaciones de que el proyecto de tesis se diseñó a principios de septiembre. Posteriormente, se solicitó la beca que fue concedida de forma oficial al quien suscribe este artículo el 21 de noviembre de 1997. El proyec- 
to de tesis fue aprobado en consejo de departamento del Departamento de Ciencias de la Información de la Facultad de Periodismo de la Universidad de La Laguna con fecha del 5 de diciembre de 1997. La incorporación al gabinete se llevó a cabo de forma oficial del uno de enero de 1998, aunque de forma efectiva ésta tuvo lugar el 7 de enero. Quedaba así demostrado que quien suscribe este artículo fue al CSIC como parte de un proyecto de investigación que, a través de la metodología de observación participante, pretendía estudiar cómo funcionan los gabinetes de prensa como fuentes periodísticas y, en concreto, los gabinetes de prensa científicos.

Conociendo a fondo la principal fuente de noticias científicas del país el CSIC-, los resultados de su investigación podrían ser generalizados, al menos en parte, al resto de las instituciones. En caso de no ser posible la generalización, al menos quedaría explicado gran parte de los mecanismos de producción de información científica en España.

Wolf establecía que el plan, aunque premeditado, no debía incluir una imposición rígida de los contenidos y McCall y Simmonds consideraban necesario una apertura en la dirección del estudio a realizar.

El plan prediseñado por nosotros seguía al pie de la letra las recomendaciones de McCall y Simmonds:

1. Debe disponerse de una cantidad de interacción social significativa en el campo al que pertenece el objeto de estudio. Estar en el gabinete durante los primeros seis meses de 1998 me permitió observar desde cómo se diseñó el plan de comunicación cuando ocurrió el desastre ecológico de Doñana, hasta participar en el proceso de producción de una nota o un dossier informativo que luego se enviaba a los medios. Además, el periodo de estancia de seis meses era suficientemente largo como para estudiar todo el proceso sin las interferencias derivadas de una estancia corta (una o dos semanas). Un defecto, este último criticado por Schlesinger, quien recomendaba estancias suficientemente prolongadas.

2. El investigador ha de realizar observaciones de hechos conexos. Durante ese periodo también pude observar no sólo cómo se genera el proceso de producción de una información del CSIC, sino que también participé en actos colaterales como organización de cenas, comidas o, incluso, en el premio de periodismo científico del CSIC en su convocatoria de 1998. Debo reconocer que estos hechos conexos al principal definen de una forma más precisa el segundo.

3. Debe realizarse algunas entrevistas formales y muchas informales. Recomendación ésta que también suscribe Wolf. En este apartado, mi estancia en el campo de investigación me permitió conversar, discutir e indagar información con muchos integrantes del CSIC. Obtuve información en conversaciones con el jefe de prensa, con los periodistas del gabinete y con numeroso personal del CSIC que no mencionare explícitamente para no perjudicarlo, pero que, sin tener grandes cargos, llevan toda una vida en la institución científica y no sólo tienen acceso a 
mucha información sino que constituyen verdaderos archivos andantes. Asimismo tuve la oportunidad de hablar con todo tipo de investigadores. Unas veces debido a mis obligaciones en el gabinete de prensa del CSIC; otras, en el ejercicio de mis labores de investigación. Debo confesar que ésta es la parte más ingrata del trabajo. La gente, que debo decirlo aquí, en el caso del CSIC tiene una categoría profesional y humana muy elevada, habla sin tapujos porque piensan que eres un «becario explotado» igual que ellos. Pero luego, tú debes anotar todas las conversaciones y los datos para, una vez seleccionados, publicar alguno de ellos. No he encontrado en la bibliografía nada acerca de cómo afronta el investigador este doble papel. Aquí también he de recomendar como uno de los mejores sitios para llevar a cabo entrevistas informales el comedor general del CSIC. Una comida distendida es el mejor sitio para indagar acerca de los porqués de muchas de los actos que uno percibe en el sistema.

4. También era necesario recopilar la mayor cantidad posible de documentos. En este sentido, debo señalar que mi trabajo como becario, una de cuyas funciones consistía en fotocopiar todo documento que pasara por el gabinete de prensa, me facilito especialmente esta labor.

5. Apertura en la dirección que toma el estudio. Aunque nuestro propósito era descubrir cómo funciona por dentro la mayor fuente de noticias científicas españolas, debo señalar que el plan consistía, principalmente, en observar todo lo que pasara, recopilar la mayor cantidad de documentos y datos posibles y, posteriormente, clasificar y desechar lo que creyéramos oportuno. Este aspecto es fundamental, pues mucho material que consideré relevante al principio, finalmente no fue utilizado; y al contrario, documentos que recopilé por simple afán de investigador, resultaron trascendentales.

Respecto a los fallos que pueden presentarse en esta metodología, descritos principalmente por Wolf y Schlesinger, pienso que tomamos todas las medidas necesarias para evitarlos. Las más importantes fueron:

a) La fase de observación estuvo siempre ligada a una de las hipótesis de investigación. (En España deben existir muchas disfunciones en el proceso de producción de información científica que provoca que la comunicación social de la ciencia sea imprecisa y con escasas fuentes).

b) Esta observación siempre estuvo orientada según principios teóricos precisos en cuanto a su metodología. La observación nunca se realizó de forma indiferenciada ni casual.

c) Tampoco se impuso desde el principio una selección rígida de los contenidos.

d) Respecto al fenómeno de socialización que se da en el investigador el «going native», según Wolf-que impide distanciarse del proceso, considero que en este caso, que siempre tuve claro que no quería quedarme a trabajar en ese gabinete, no se produjo. 
e) La desconexión con el sistema en estudio -el «disengagement» de Schlesinger- se produjo en agosto de 1998, época en la que comenzó la reestructuración del material recogido. Hasta el momento de redactar la investigación, en octubre de 1999, había pasado suficiente tiempo como para valorar los hechos con la perspectiva adecuada.

f) El error más significativo de la metodología, derivado de llegar al sistema en estudio en etapas posteriores al comienzo real del proceso de producción, lo que provoca situaciones de automatismo que enmascaran las relaciones reales entre los agentes participantes en los hechos, quedó, a nuestro juicio, superado. La corrección de tal error, mediante la observación de situaciones de crisis, fue posible gracias a que durante el periodo de observación enero-julio de 1998, se produjo el episodio de crisis informativa más grave ocurrido en la historia del CSIC: el accidente ecológico de Doñana. También hubo otros episodios menores, como las reiteradas manifestaciones de los científicos jóvenes españoles en busca de mejores expectativas laborales.

\section{RESULTADOS TRAS LA APLICACIÓN DE LA METODOLOGÍA}

Una vez aplicada la metodología de «Observación Participante» se obtuvieron los siguientes resultados respecto del gabinete de prensa del CSIC como emisor de información mediática:

1. Como organización, los gabinetes de prensa están fuertemente jerarquizados. Sin embargo, en el caso del CSIC, la jerarquización es mucho más férrea de lo que cabría esperar para un organismo científico. No obstante, debe matizarse que el presidente del CSIC es un cargo político elegido por el ministro de turno responsable del área científica. Es decir, que aunque el CSIC sea un organismo científico, no posee la autonomía de una universidad pública respecto a la difusión de información veraz hacia los medios. El CSIC tiene las restricciones de las organizaciones vinculadas con el poder político.

2. Esto implica, entre otras consecuencias, que la selección de los periodistas que conforman el gabinete de prensa no se haga en función de criterios de mérito y capacidad, sino que priman criterios como la socialización con los jefes y, sobre todo, con los políticos que ejercen el poder. Es decir, que para trabajar en estos gabinetes se tiene más en cuenta elementos como la confianza (ya sea por amistad o por tener o haber tenido familiares en el organismo) que la capacidad.

3. Esto es importante porque indica que el periodista de gabinete se debe a la fuente que lo contrata. Si se pierde la confianza entre fuente y periodista de gabinete se suspende la vinculación laboral o se le destina a otras funciones alejadas con el contacto directo con periodistas de los medios. 
4. La selección de la noticia que el gabinete emite como fuente jamás se hace en función de los intereses de la sociedad. Siempre prima el interés de la fuente, nunca del periodista de los medios. El periodista de gabinete utiliza los conocimientos de periodismo aprendidos en la facultad para «traducir» al lenguaje periodístico las ideas que la fuente quiere que sean difundidas. Se facilita así su publicación pues como cumplen los criterios exigidos por los medios, tiene más posibilidades de ser seleccionadas. De esta manera, se refuerzan las noticias positivas para la fuente y se ocultan o minimizan (en el caso de que los periodistas se hayan enterado por otros medios) las noticias negativas o de conflicto hacia la fuente.

5. Esto provoca que el trabajo del «periodista» de gabinete de prensa sea más el de un propagandista que el de un informador periodístico, intentado vender como noticia lo que en la mayoría de los casos no es sino publicidad. Por eso es tan preocupante el dato de que cada día los medios publican más información inducida por los gabinetes de prensa. Y es que esas noticias no son información relevante para la sociedad, sino importantes para la fuente. El lector cree que el periodista ha seleccionado esa noticia en función de todas las que ha investigado y que la elegida es la que cumple mejor los criterios de noticiabilidad. Sin embargo, la realidad es que el periodista elige, usando criterios de noticiabilidad, pero de entre las que le llegan de los gabinetes de prensa y, por tanto, todas esas son interesadas.

6. El periodista de los medios contrasta pocas veces lo que le llega de un gabinete de prensa oficial. Es lo que Furio Colombo denomina «noticia acatamiento». Es decir, noticias que llegan desde la fuente con plus de credibilidad tal que el periodista piensa que no es necesario contrastarlas. Si se alcanza esta situación es cuando el gabinete de prensa logra su efectividad máxima porque tiene la posibilidad de introducir noticias falsas. «Esta circunstancia quedó demostrada en el episodio de crisis informativa tras la catástrofe ecologica de Doñana en 1998. Ningún periodista cuestionó la información que procedía del CSIC como fuente (Elías, 2001b)».

7. El gabinete de prensa ejerce otra de las formulas del antiperiodismo: puede actuar como censor. Es decir, puede prohibirle a una fuente que hable con un periodista si esas declaraciones hacen daño a la institución. También actúa como censor cuando interpone dificultades entre el periodista y la fuente, evitando que ambos entren en contacto. Actúa también como censor cuando «adiestra» a la fuente sobre lo que le conviene contestar al periodista y lo que le conviene callar. Pese a todas estas actitudes, resulta curioso que el periodista de gabinete de prensa no tenga «mala conciencia» ética ni profesional cuando utiliza sus conocimientos periodísticos no para facilitar la información a la sociedad sino para lo contrario: intoxicar a los medios con informaciones positivas para la fuente pero poco relevantes, al 
tiempo que evita otras que no interesan. Tampoco se percibe desde la sociedad animadversión hacia la profesión de periodista de gabinete de prensa. En cualquier caso, no despierta los recelos de los antiguos censores franquistas. Sin embargo, en muchas ocasiones hacen un trabajo similar.

8. El periodista de los medios tiene que luchar mucho más para obtener la verdad de la fuente si existe un gabinete de prensa que adiestra a las fuentes y se interpone entre ambos cuando interesa que una noticia no trascienda. La perjudicada es la sociedad que se queda sin saber la verdad.

9. La eficiencia de un gabinete de prensa se mide en función de dos criterios deontológicamente reprochables: el número y espacio de noticias positivas (no necesariamente verdaderas) que inserta en los medios de comunicación. El otro factor que mide la eficiencia es el del número de noticias (verdaderas) pero negativas que logró evitar que llegara a los medios, así como el número de noticias negativas en las que logró minimizar su repercusión en prensa. Esta eficiencia siempre es buena para la fuente pero muy mala para el periodismo y, por extensión, para la sociedad.

\section{EVALUACIÓN DE LA APLICACIÓN \\ DE LA «OBSERVACIÓN PARTICIPANTE» AL CASO DE LOS GABINETES DE PRENSA COMO FUENTES}

Una vez aplicada la metodología y vistos los resultados se observó lo siguiente respecto a la evaluación de la aplicación metodológica:

1. Como se ha mencionado, el comportamiento ético, respecto a la deontología periodística, de los que trabajan en los gabinetes de prensa, es bastante reprochable. Los temas que se tocan son muy delicados desde el punto de vista político. Sobre todo si es un gabinete como el del CSIC que pertenece al Gobierno y que controla la mayoría de la información científica de España. Esto provoca que si se utilizara una metodología cualitativa basada en la entrevista, difícilmente los periodistas de gabinetes de prensa investigados afirmarían que ellos manipulan, tergiversan o censuran información y fuentes. El proceso de selección para su trabajo, en el que prima la socialización con los jefes frente a los méritos objetivos y capacidades, también provoca que su visión del asunto sea demasiado subjetiva o que no digan la verdad por miedo a perder la confianza de los jefes y, por extensión, perder sus trabajos. Resulta, además, muy complejo utilizar entrevistas en las que puedan detectarse las falsedades. Por otra parte, la entrevista, aunque sea en profundidad y no dirigida, no capta procesos ni manera de hacer determinados trabajos sino cómo el entrevistado cree que se hacen o deben hacerse. 
2. Si se hubiese aplicado otra metodología los resultados hubiesen cambiado drásticamente. De hecho, antes de comenzar la investigación nosotros pensábamos que la existencia de un gabinete de prensa era algo positivo para una organización científica. Teníamos esta hipótesis tras consultar los estudios existentes en España sobre gabinetes de prensa. No olvidemos que existe mucha investigación publicada en la que se concluye que la existencia de gabinetes es algo positivo para el periodismo. Es decir, justo lo contrario de lo que se afirma tras nuestra investigación. Todas esos estudios que alaban a los gabinetes están elaborados con metodología de entrevista. No obstante, en otros estudios referidos a fuentes periodísticas interesadas, la demostración de la manipulación la suele hacer el periodista (o el investigador) de los medios tras contrastar la información que dieron los gabinetes con la que, pasado un tiempo, se obtiene del hecho noticioso. Pero, obviamente, es complejo obtener esos resultados desde las propias fuentes (los gabinetes de prensa) que siempre se definen como los «amigos de los periodistas», los que «facilitan el trabajo periodístico» y los que «propician que la sociedad conozca informaciones que de otra manera no saldrían a la luz». Además no siempre, y menos en los casos de las ciencias experimentales, se sigue el hecho una vez que ha dejado de ser noticia.

3. La observación participante permitió observar cómo se manipula la información en el caso de una gran noticia de carácter nacional y con repercusiones políticas: la catástrofe ecológica de Doñana. Este episodio de crisis informativa en el que intervino el CSIC duró varios meses, por lo que sólo se podía estudiar, desde dentro, mediante la metodología de Observación Participante.

\section{CONCLUSIÓN FINAL}

Con todas estas observaciones considero que se han llevado a cabo todos los controles necesarios para que la metodología de «Observación Participante» estuviera bien aplicada y que, por tanto, los resultados sean concluyentes. No obstante, sería interesante que se aplicaran estos pasos a otras fuentes periodísticas: desde gabinetes de prensa científicos como los de la NASA, las revistas Nature o Science, hasta los de comunidades autónomas, gobierno, ministerios, universidades o parlamento europeo, entre otras muchas instituciones. También a los gabinetes de comunicación de empresas privadas, sobre todo de multinacionales de la energía, la comunicación o la alimentación. Otro sistema de estudio en el que debería aplicarse esta metodología es en los propios medios de comunicación: periódicos, televisiones $\mathrm{y}$ emisoras de radio para descubrir cómo funcionan por dentro. Asimismo, y debido a la mayor relevancia que adquieren cada día los programas de la denominada «telebasura», sería conveniente aplicar esta metodología a las productoras de televisión encargadas de realizar estos programas. 


\section{REFERENCIAS BIBLIOGRÁFICAS}

BECKER, H. S. y GEER, B. (1958): «Participant observation and interviewing: a rejonder», Human Organization, $\mathrm{n} .^{\circ} 17$.

Blumer, H. (1954): «What is Wrong with Social Theory», American Sociological Review, $\mathbf{n}{ }^{\circ} 19$.

ELIAS, C. (2001a): «Estudio cuantitativo de las fuentes en el periodismo español especializado en ciencia». Revista Latina de Comunicación Social (revista electrónica), ${ }^{\circ} 38$ (febrero).

- (2001b): «Periodismo especializado en medio ambiente: el caso Doñana como paradigma de manipulación informativa». Ámbitos, Revista Internacional de Comunicación, n. ${ }^{\circ}$ 6, pp. 279-303.

Ellot, P. (1972): The Making of a Television Series. A Case Study in the Production of Culture. London. Ed. Contestable.

Grrun, T. (1978): «Media Sociology, the Dominant Paradigm». Theory and Society, n. ${ }^{\circ} 6$.

JANKOWSKY, N. W., y WESTER, F. (1993): «La tradición cualitativa en la investigación sobre las ciencias sociales: contribuciones a la investigación sobre la comunicación de masas», en Jensen K. B. y Jankowsky (1993), Metodologías cualitativas de investigación en comunicación de masas. Barcelona. Bosch-Comunicación.

Johnson, J. M. (1975): Doing Field Research. New York. Free Press.

MCCALL, G. J. y Simmons, J. L. (1969): Issues in Participant Observation: a Text and Reader. Massachussets Ed. Addison-Wesley.

Ramírez, T. (1995): Gabinetes de comunicación. Barcelona. Bosch-Comunicación.

Schatzman, L., y Strauss, A. L. (1971): Field Research: Strategies for a National Sociology, New Jersey. Ed. Englewood Clift.

SCHLESINGER, P. (1978): «Between Sociology and Jounalism. Some Reflections on a Newsroom Ethnography», ponencia para la «Sociological Review Monograph on Jouurnalism and the Press».

WOLF, M. (1996): La investigación en la comunicación de masas: críticas y perspectivas. Barcelona. Paidos. 